\title{
Las relaciones cognitivas y conductuales en las interacciones didácticas
}

\section{Relationship between behavioral and cognitive categories in didactic interactions}

\section{ANGELA FigueroA IBÉRICO*}

El propósito de este estudio es identificar y analizar las interacciones del docente y los estudiantes en las prácticas pedagógicas orientadas a un aprendizaje por competencias. Para ello, desarrollamos una investigación cualitativa basada en el estudio exploratorio de casos en dos instituciones educativas públicas de la ciudad de Lima, Perú. La muestra está constituida por 16 docentes y 440 estudiantes de secundaria. Realizamos observaciones de 32 sesiones de clases y entrevistamos a los docentes; asimismo, desarrollamos un análisis de contenido a partir de un sistema de categorías vinculadas al proceso cognitivo (Cuadrado y Fernández, 2008) y un sistema de categorías propuestas para la conducta (Velasco, 2007), cuyo objeto de estudio son las orientaciones pedagógicas para el desarrollo del aprendizaje por competencias (Ministerio de Educación del Perú, 2016). Los resultados demuestran la complementariedad e interdependencia de las categorías cognitivas y conductuales, y evidencian que en las prácticas pedagógicas la cognición tiene mayor peso que la conducta.

The purpose of this study is to identify and analyze the interactions of the teacher and students in pedagogical practices aimed at competency-based learning. A qualitative research was conducted based on an exploratory study of cases in two public educational institutions in the city of Lima. The sample included 16 teachers and 440 high school students. Direct observations were carried out during 32 classes, where teachers were also interviewed. A content analysis was developed considering a system of categories linked to the cognitive process (Cuadrado \& Fernández, 2008) as well as a system of categories proposed for behavior (Velasco, 2007), having as subject of study the pedagogic orientations for the development of competencies put forward by de Ministry of Education of Perú (2016). The results demonstrate the complementarity and interdependence of the cognitive and behavioral categories, revealing that in pedagogical practices, cognition has more weight than behavior.

\section{Palabras clave:}

interacciones didácticas, categorías cognitivas, categorías conductuales, aprendizaje por competencias

\section{Keywords:}

didactic interactions, cognitive categories, behavioral categories, competency-based learning

Recibido: 21 de febrero de 2018. | Aceptado para su publicación: 31 de julio de 2018. DOI: 10.31391/S2007-7033(2018)0051-006

\footnotetext{
*Magíster en Didáctica, Teorías de la Enseñanza y Prácticas Escolares. Investigadora del Instituto de Investigación y Políticas Educativas de la Universidad Antonio Ruiz de Montoya, Lima, Perú. Sus líneas de investigación versan sobre la didáctica en las ciencias sociales, la educación en contextos rurales y las representaciones docentes.
} 


\section{INTRODUCCIÓN}

$1 \mathrm{n}$ las tres últimas décadas se ha consolidado la reflexión teórica y empírica

4 que sustenta el valor de las interacciones didácticas para explicar la relación entre el conocimiento, la conducta y el aprendizaje en la comprensión de las prácticas pedagógicas. Los estudios que abordan las interacciones, desde los aprendizajes de los estudiantes y su relación con las formas de enseñanza de los docentes, revelan que estos aspectos son centrales en el proceso educativo (Mares, Guevara, Rueda, Rivas y Rocha, 2004) y están presentes en las diversas tradiciones de la investigación (Villalta y Martinic, 2009). En consecuencia, contamos con un apreciable número de estudios que han sido analizados desde diferentes dimensiones, estructuras de participación y unidades de análisis (Coll y Sánchez, 2008) y desde diversos contextos educativos, niveles de enseñanza y disciplinas o áreas curriculares.

De esta forma, aproximarse al estudio de las condiciones reales en una sala de clase nos invita a conocer de cerca la continuidad en la interacción del docente y los estudiantes en el proceso de enseñanza y aprendizaje. Sin embargo, algunos diagnósticos advierten situaciones preocupantes en los estilos de la práctica pedagógica: predominancia de una pedagogía directiva con poco diálogo y conexión con los estudiantes (Martinic, Vergara y Huepe, 2013) y desarrollo de interacciones que no resultan estimulantes para construir aprendizajes (González, Eguren y De Belaúnde, 2017). A pesar de los esfuerzos desde diversos frentes, persisten evidencias de situaciones tradicionales de inequidad en las interacciones, con una escasa presencia del diálogo y una mayor frecuencia de intervenciones docentes de carácter autoritario (Velasco y Alonso, 2008).

En el Perú, el documento marco de la política educativa de la educación básica se define como Currículo Nacional ( $\mathrm{CN}$ ), el cual propone un enfoque del aprendizaje por competencias que orienta la práctica pedagógica en los niveles de inicial, primaria y secundaria en sus diferentes modalidades. Este documento define la competencia como "la facultad que tiene una persona de combinar un conjunto de capacidades a fin de lograr un propósito específico en una situación determinada, actuando de manera pertinente y con sentido ético" (Ministerio de Educación del Perú [Minedu], 2016, p. 29), y describe la capacidad como los recursos (conocimientos, habilidades y actitudes) que los estudiantes utilizan de un modo articulado para afrontar una situación (Minedu, 2016).

Anterior al CN, existió otro documento orientador denominado Diseño Curricular Nacional (DCN), que contenía un enfoque por competencias; según Tapia y Cueto (2017), este proponía un planteamiento fragmentado de las competencias como capacidades, conocimientos y actitudes, que sugería que desarrollar una competencia significaba lograr cada una de las "partes". Este proceso de cambio ha evidenciado algunas confusiones docentes. En un estudio sobre la implementación del nuevo CN, Guerrero (2018) advierte que "los docentes creen que no hay conocimientos en el CN porque ya no los ven en forma de un listado de temas, como estaban acostumbrados a hacerlo en el DCN" (p. 83). Ante ese diagnóstico, la autora aclara que la propuesta del CN no señala que los conocimientos no existan o no sean importantes, sino que la competencia articula recursos, uno de los cuales son los conocimientos, y estos conocimientos están en relación con otros recursos y en contextos específicos.

En este escenario educativo surgen dos cuestiones orientadoras: ¿cómo interactúan el docente y los estudiantes en el aula? y ¿cómo esas interacciones evidencian una práctica 
pedagógica enfocada hacia un aprendizaje por competencias? De esta forma, nuestro estudio se propone identificar y analizar las interacciones del docente y los estudiantes en las prácticas pedagógicas orientadas a un aprendizaje por competencias.

\section{MARCO TEÓRICO}

Los enfoques socioculturales y sociolingüísticos en el estudio de las interacciones

Existe un aporte significativo de estudios que se basan en la teoría sociocultural o de la actividad del psicólogo soviético Vygotsky para comprender que las prácticas pedagógicas ponen en evidencia la interacción como un espacio intersubjetivo que hace posible la comunicación y el desarrollo del pensamiento (Rogoff, 1993). Desde este enfoque, el lenguaje aparece como la herramienta que promueve el pensamiento y con la cual se comunican y construyen significados de manera conjunta en el aula (Forero-Sáenz, 2008) y el aprendizaje supone la participación del sujeto en actividades culturalmente organizadas (Rogoff, 1993) e inseparables del contexto donde se adquieren y utilizan (Coll y Sánchez, 2008).

Eligiendo como contexto educativo el aula, un espacio organizado en forma intencional para propiciar el proceso de enseñanza-aprendizaje, comprendemos que los factores de la interacción didáctica que se producen en ella no son siempre transferibles a otros contextos educativos. Esto se debe a las diferencias entre el contexto físico - características espaciales, infraestructura, materiales- y el contexto mental - representaciones, expectativas, afectos, motivaciones- (Coll y Sánchez, 2008), que inciden en que ambos contextos son espacios mediadores por excelencia de la cultura (Villalta, Assael y Martinic, 2013). Así, el aula y las prácticas pedagógicas asumen una relación de interdependencia, en la cual interesa conocer cómo se movilizan los comportamientos comunicativos interactivos en el proceso de enseñanza y aprendizaje desde un análisis del discurso didáctico.

Las interacciones didácticas son prácticas comunicativas que se desarrollan en el aula, entre la acción intencionada de enseñanza por parte del docente con las intervenciones consecuentes y emergentes de los estudiantes para aprender (Velasco, 2007; Villalta y Martinic, 2013), y son construcciones culturales, porque incluyen significados compartidos que sintetizan las diversas culturas que asimilan los actores en diversos contextos (Villalta, Martinic y Guzmán, 2011). En esos contextos, docentes y estudiantes usan códigos y siguen criterios compartidos para construir el conocimiento, y discuten y argumentan a través de reglas implicadas en la producción del discurso didáctico (Cubero et al., 2008).

La consolidación del enfoque sociocultural para analizar las interacciones didácticas va de la mano con los cambios en la delimitación del concepto de didáctica. La didáctica se aleja de la concepción de herramienta, estrategia o técnica para la transmisión de saberes y se concibe como una construcción cultural de competencias comunicativas capaces de hacer pertinentes los saberes curriculares a los contextos construidos en la interacción (Díaz y Ochoa, 2007).

En el análisis de la interacción didáctica, según Velasco (2007), es importante destacar la relación entre dos dimensiones diferentes en acciones conjuntas: la dimensión cognitiva, independientemente de lo que sea observable en cuanto a lo que el sujeto 
haga o deje de hacer de los procesos de pensamiento; y la dimensión comportamental o de la conducta, referida a los procesos observables de manera empírica que ocurren en un sujeto, sin tener en cuenta lo cognitivo. Consideramos que, en la interacción, dos o más sujetos entran en contacto e intercambian información mediante acciones (cogniciones y conductas) mutuamente referentes e interdependientes; la conversación es la herramienta de apropiación y desarrollo del conocimiento.

Desde del enfoque sociolingüístico, entendemos que la comunicación es la expresión cultural más elaborada y estable en la constitución de significados (Villalta y Martinic, 2009) que necesita y construye un contexto que ofrece elementos para que se desarrollen competencias comunicativas. En ese sentido, resaltan las competencias comunicativas del docente porque están estrechamente relacionadas con la construcción de climas favorables en el aula (Castellá, Comelles, Cros y Vilá, 2007; Minedu, 2012).

Asumiendo que la interacción se hace posible por la comunicación intencional en el aula, es preciso aproximarnos a un análisis del discurso didáctico, entendido como un intercambio secuencial entre sujetos y situaciones referentes que se requiere fraccionar. Para Sánchez et al. (2008), la unidad de análisis más inclusiva corresponde a la unidad curricular (por ejemplo, un tema en una asignatura), y dentro de esa unidad curricular, cabe hablar de diferentes sesiones, delimitadas institucionalmente a través de un horario.

Las sesiones, según los autores, se componen, a su vez, de actividades típicas de aula y cada una de estas puede integrar diversos episodios, los cuales, según Cuadrado y Fernández (2008), son definidos como un conjunto de intercambios comunicativos entre docente y estudiantes, o entre estudiantes, dirigidos a alcanzar un acuerdo sobre una cuestión concreta relacionada con el aprendizaje.

\section{Un enfoque por competencias desde la escuela}

Los cambios que han generado los sistemas productivos como resultado de los procesos de globalización han afectado de modo directo la economía, el trabajo y, por ende, los sistemas educativos. En ese contexto, desde mediados de la década de los noventa, la introducción del enfoque por competencias en el actual proceso reformador de los sistemas educativos ha incorporado nuevos paradigmas y "ha inspirado una reescritura de programas" (Perrenoud, 2008, p. 2), en la concepción del aprendizaje y, en consecuencia, en el diseño curricular.

Conceptualizar el término competencias, en el análisis de Díaz (2006), supone reconocer la combinación de tres elementos indispensables: el dominio de una información, el desarrollo de una habilidad o de una serie de habilidades, y una situación problema o situación real inédita. Esta noción se complementa cuando Perrenoud (2008) plantea que la competencia es el saber movilizar un conjunto de recursos para adaptarse a una situación, sobre todo en la acción.

Refiriéndonos al rol del docente, en una entrevista el profesor Lessard, investigador de la Universidad de Montreal, afirma que el enfoque por competencias se aleja de dos identidades tradicionalmente fuertes: primero, la identidad del maestro instruido que "simboliza al profesor de secundaria, que se concibe como la persona 
que tiene una aptitud, que dispone de un conocimiento que él mismo transmite y para quien la enseñanza consiste en desarrollar y transmitir ante sus alumnos todo ese texto del saber", y segundo, la del maestro artesano, es decir, aquel que "es capaz de enseñar porque ha visto a otros maestros hacerlo, porque ha observado a sus compañeros y así ha ido construyendo el proceso de socialización profesional e interiorizando el 'habitus' dominante del profesor" (Luengo, Luzón y Torres, 2008, p. 10). Ante estos dos modelos docentes, Lessard hace hincapié en que el profesional competente debe ser un gran conocedor de su disciplina, sensibilizarse con su cultura y ser capaz de reflexionar sobre su propia práctica.

El enfoque por competencias presenta retos porque solo podrá mostrar su mayor riqueza si se logra incorporar de manera real en la tarea docente. Díaz (2006) advierte que es necesario clarificar su propia propuesta, lo cual significa construir un lenguaje que contenga tanto su propuesta como sus límites y, a la par, explorar con mayor cuidado las dimensiones pedagógicas de un tema, que evidentemente reanuda una discusión sobre el sentido del aprendizaje escolar, pero que la mayoría de los autores que lo abordan lo omiten o lo desconocen.

El Minedu define las competencias como facultades que tiene una persona de combinar y movilizar un conjunto de capacidades, entendidas como conocimientos, habilidades y actitudes, a fin de lograr un propósito específico en una situación determinada. El máximo órgano educativo del Estado peruano propone en el CN (2016) establecer los aprendizajes que se espera alcancen los estudiantes como resultado de su formación y propone el desarrollo de 31 competencias en el perfil del estudiante egresado, las cuales deben darse "en forma vinculada, simultánea y sostenida durante la experiencia educativa" (Minedu, 2016, p. 29). Es importante señalar que esas competencias se disgregan en capacidades y, a su vez, en una secuencia de estándares que describen los grados de complejidad en los tres niveles de educación.

\section{Metodología}

Para responder al objetivo de este trabajo, optamos por una investigación de tipo cualitativa, basada en el estudio exploratorio de casos. La recolección de datos fue un proceso que duró cuatro meses (de abril a agosto de 2016) y se desarrolló de manera secuencial: primero a través de las filmaciones y después mediante la entrevista a cada docente. Las filmaciones se llevaron a cabo en dos sesiones de clases por docente, de noventa minutos cada una, con ayuda de una videocámara. Las entrevistas fueron presenciales, abiertas y orientadas con una guía de preguntas y el soporte de una grabadora; las realizamos un mes después de las filmaciones con la intención de que el docente entrevistado pudiera introducir nuevos temas de conversación que permitieran completar o precisar las interpretaciones en la investigación. En ambas técnicas, usamos guías de observación para registrar información complementaria al estudio.

Las dos instituciones educativas públicas que formaron parte de la investigación presentan similitud en las siguientes características: son de modalidad presencial escolarizada, pertenecientes a la Unidad de Gestión Educativa Local 2; cuentan con infraestructura escolar básica; la población estudiantil es mixta (hombres y mujeres) y relativamente homogénea en términos de nivel socioeconómico; la planta docente 
está completa, tienen directores y subdirectores, y reciben con regularidad los recursos y materiales educativos del Minedu (como programas de capacitación y textos escolares). Ambas instituciones se ubican en distritos correspondientes a la región norte de la ciudad de Lima (Los Olivos y San Martín de Porres), cuyas actividades económicas principales son el comercio y la industria.

La muestra estuvo conformada por 232 estudiantes y 8 docentes en la primera institución educativa, y por 208 estudiantes y 8 docentes en la segunda institución. Los estudiantes tenían entre doce y diecisiete años de edad, y estaban distribuidos en los cinco años del nivel de secundaria. Todos los docentes tienen formación superior, cuentan con más de cinco años de experiencia profesional y pertenecen a diversas áreas curriculares (matemática, comunicación, ciencia, tecnología y ambiente, historia, geografía y economía, religión, arte y tutoría) de secundaria. La participación docente fue voluntaria y aceptaron un protocolo de consentimiento informado.

Para alcanzar el objetivo de nuestro trabajo, tomamos como objeto de estudio las diez orientaciones pedagógicas propuestas para el desarrollo de competencias en los estudiantes del Currículo Nacional de la Educación Básica del Minedu (2016). Según el actual enfoque pedagógico, se han definido estas orientaciones enmarcadas en las corrientes socioconstructivistas del aprendizaje para ser consideradas por los docentes en la planificación, ejecución y evaluación de los procesos de enseñanza y aprendizaje en los contextos educativos donde se desenvuelven.

Esquema. Orientaciones pedagógicas para el desarrollo de competencias

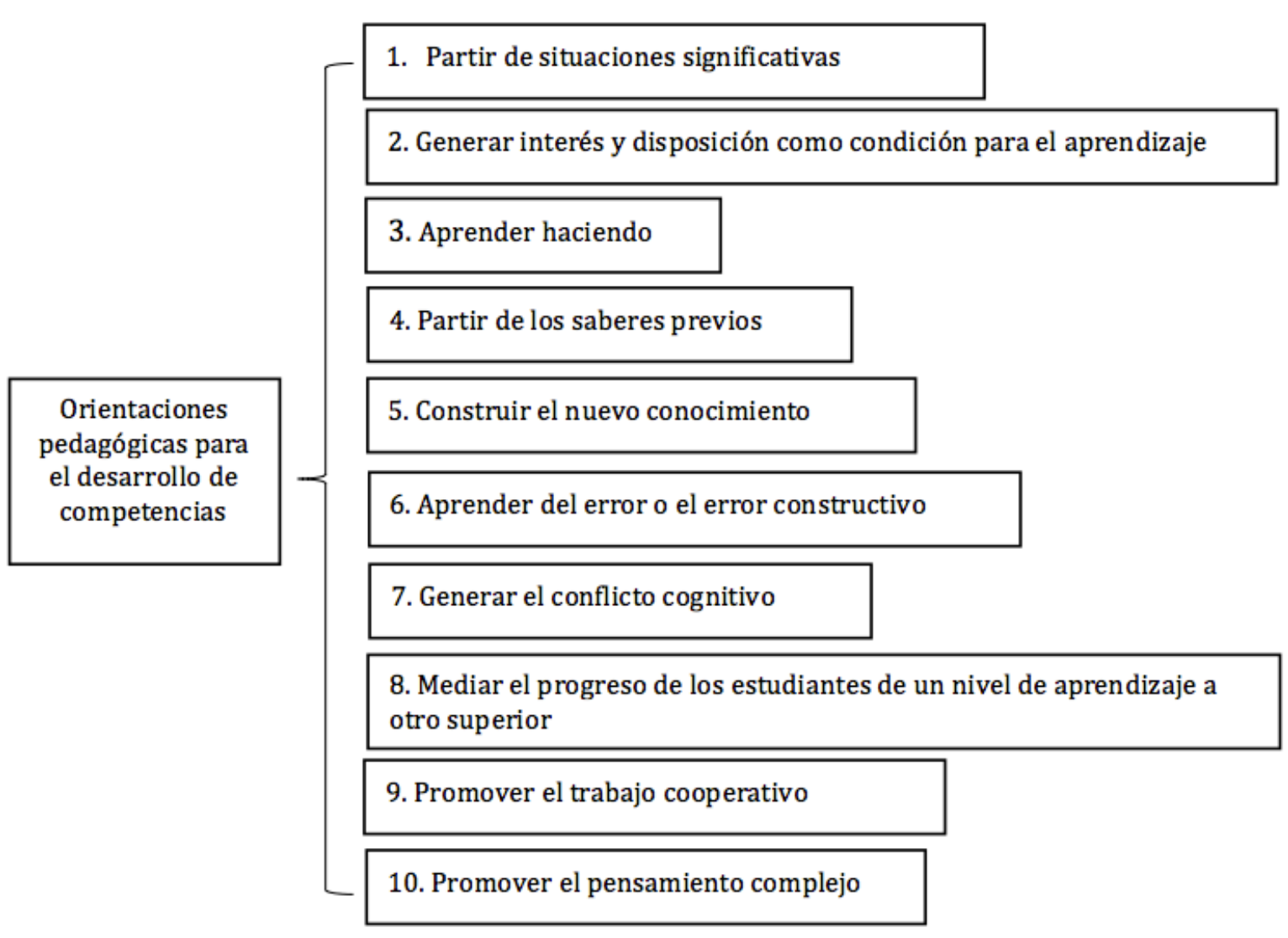

Fuente: Minedu (2016). 
Para el análisis de los resultados, se seleccionaron dos sistemas de categorías: las interacciones cognitivas del estudio de Cuadrado y Fernández (2008), que presenta diez categorías sobre los recursos comunicativos verbales y no verbales de los profesores, y las categorías propuestas para la conducta del alumno y del docente (Velasco, 2007), ambas vinculadas al logro de aprendizaje de los alumnos.

Tabla 1. Categorías de análisis vinculadas al proceso cognitivo

\begin{tabular}{|c|c|}
\hline Categoría & Descripción \\
\hline $\begin{array}{l}\text { Marco social de } \\
\text { referencia }\end{array}$ & $\begin{array}{l}\text { Comentarios relacionados con experiencias o vivencias personales que profesores y } \\
\text { alumnos han adquirido fuera del contexto escolar y que en el momento de su exposi- } \\
\text { ción guardan una vinculación directa con los contenidos que se trabajan en clase }\end{array}$ \\
\hline $\begin{array}{l}\text { Marco específico de } \\
\quad \text { referencia }\end{array}$ & $\begin{array}{l}\text { Referencias a contenidos anteriores que guardan relación con los nuevos que se } \\
\text { trabajan y que permiten mostrar la contextualización y continuidad de los contenidos } \\
\text { curriculares }\end{array}$ \\
\hline $\begin{array}{l}\text { Formulación de } \\
\text { preguntas }\end{array}$ & $\begin{array}{l}\text { Interrogantes que se formulan para acceder a los conocimientos previos del alumnado } \\
\text { y comprobar los significados que van construyendo }\end{array}$ \\
\hline Metaenunciados & $\begin{array}{l}\text { Expresiones contextualizadoras que informan a los interlocutores de las acciones que } \\
\text { se van a emprender o se están llevando a cabo para facilitar la comprensión de las } \\
\text { tareas }\end{array}$ \\
\hline Repeticiones literales & Reproducción literal de las aportaciones de los alumnos \\
\hline Reformulaciones & $\begin{array}{l}\text { Forma de recontextualizar las intervenciones del alumno y acentuar aquellas partes o } \\
\text { enunciados que se consideran válidos, o bien, corregir los errores identificados o las } \\
\text { respuestas incompletas }\end{array}$ \\
\hline Reelaboraciones & $\begin{array}{l}\text { Reconstrucción de las aportaciones de los alumnos dirigidas a corregir errores estruc- } \\
\text { turales o gramaticales; reorientar esas aportaciones conforme a la línea argumentativa } \\
\text { que se sigue en clase; compilar las aportaciones de varios participantes y extraer la } \\
\text { idea o concepto general que las engloba; destacar determinados aspectos de interés; } \\
\text { evidenciar las relaciones existentes entre diferentes significados, etcétera }\end{array}$ \\
\hline Autorreformulaciones & $\begin{array}{l}\text { Reformulación de las expresiones del docente para facilitar su comprensión o atraer la } \\
\text { atención del alumnado }\end{array}$ \\
\hline Resúmenes & Recapitulación de las informaciones dadas o de las acciones llevadas a cabo \\
\hline Gestos ilustrativos & $\begin{array}{l}\text { Manifestación de comportamientos comunicativos no verbales dirigidos a facilitar la } \\
\text { decodificación de los mensajes verbales }\end{array}$ \\
\hline
\end{tabular}

Fuente: Cuadrado y Fernández (2008).

Figueroa. Las relaciones cognitivas y conductuales en las interacciones didácticas Sinéctica 51 www.sinectica.iteso.mx 
Tabla 2. Categorías de análisis propuestas para la conducta

\begin{tabular}{|c|c|}
\hline Alumno & Docente \\
\hline Expresa ignorancia, perplejidad & Expresa ignorancia, perplejidad \\
\hline Niega, escucha no atenta & Consigna actividades. Regula la participación, corrige \\
\hline \multirow[t]{2}{*}{ No escucha } & Sugiere respuesta, induce a ella \\
\hline & $\begin{array}{l}\text { Requiere respuesta implícita o explícitamente } \\
\text { (pregunta directa o indirecta). Indaga, explora }\end{array}$ \\
\hline $\begin{array}{c}\text { Se explica, se justifica, formula aclaración, corrige al } \\
\text { otro (docente o alumno), construye ideas en discurso } \\
\text { propio }\end{array}$ & $\begin{array}{l}\text { Explica o justifica tema o acción. Instruye, recita, } \\
\text { expone, expresa conocimiento, responde, aclara (inclu- } \\
\text { so apoyándose en un texto o en el pizarrón) }\end{array}$ \\
\hline \multicolumn{2}{|l|}{ Responde, expresa o expone conocimiento } \\
\hline \multirow{3}{*}{$\begin{array}{l}\text { Elogia, estimula, alaba, confirma al otro (docente o } \\
\text { alumno) }\end{array}$} & $\begin{array}{l}\text { Acepta ideas, opiniones o expresiones emotivas, } \\
\text { confirma }\end{array}$ \\
\hline & Estimula, apoya, acompaña, aprueba \\
\hline & $\begin{array}{l}\text { Hace metaanálisis, sintetiza, recapitula y reubica } \\
\text { aportes }\end{array}$ \\
\hline Silencio & Silencio \\
\hline
\end{tabular}

Fuente: Velasco (2007).

Dadas las características metodológicas de la investigación, fue conveniente usar el software Atlas.ti, versión 7.5.4. Teniendo en cuenta que el procedimiento para el análisis de datos consiste en seleccionar unidades de análisis de un contexto a las cuales el investigador codifica, el programa Atlas.ti involucra un proceso de tres etapas: codificación de la información, categorización y estructuración de una o más redes de relaciones entre las categorías y estructuración de hallazgos o teorización (Varguillas, 2006).

Para el análisis de los resultados, proponemos una secuencia de acciones para identificar y analizar las relaciones en las interacciones didácticas en las diez orientaciones pedagógicas propuestas en el Currículo Nacional de la Educación Básica del Minedu (2016) para el desarrollo de competencias en los estudiantes: transcripción de los 48 documentos (32 trascripciones de las filmaciones y 16 transcripciones de los audios de entrevistas); creación de una unidad hermenéutica en el Atlas.ti, en la que se inserten los 48 documentos convertidos en un formato especial por el software; selección de los fragmentos de interacciones en los documentos que evidencien las orientaciones pedagógicas; codificación de los fragmentos según categorías cognitivas (a las cuales identificaremos como $\mathrm{Ccg}$ ) y categorías conductuales (a las cuales identificaremos como Ccd); y análisis en las relaciones de las categorías cognitivas y las categorías conductuales, identificadas en las interacciones didácticas según cada orientación pedagógica; la información se complementa con las declaraciones de los docentes en las entrevistas y la literatura especializada. 


\section{ANÁLISIS DE RESULTADOS}

A continuación describimos episodios interactivos de sesiones de clases, que evidencian las orientaciones pedagógicas enfocadas al aprendizaje por competencias; identificamos y analizamos en ellas las relaciones de las interacciones cognitivas y conductuales:

\section{Partir de situaciones significativas}

Implica diseñar o seleccionar situaciones reales o simuladas, pero factibles, que respondan a los intereses de los estudiantes, que les permitan movilizar sus saberes previos y establecer relaciones con una nueva situación, lo cual constituye un nivel de desarrollo mayor al que tenían (Minedu, 2016).

Seleccionamos un episodio de una interacción en la sesión de clase de Tutoría en primer año de secundaria:

Docente: La Organización Mundial de la Salud dice que cien mil niños fuman cigarrillos en el Perú. ¿Cuáles son las razones por las que ustedes creen que niños y adolescentes empiezan a fumar cigarrillos a tan temprana edad? Pensamos en silencio, escribamos la respuesta en el cuaderno y participamos en una puesta en común en forma ordenada. [Los alumnos escriben las respuestas en su cuaderno y en un tiempo breve, levantan la mano y participan.]

Rashid: Sus padres no les explicaron cuáles son los riesgos de fumar.

Kadir: Sienten presión del grupo y por eso empiezan a fumar.

Docente: Sienten presión del grupo.

Celso: Porque lo toman como un juego y no se dan cuenta de las consecuencias que puede tener.

Maxi: Porque quieren experimentar las cosas que hacen los mayores.

Docente: Ciertamente el descuido de los papás al no explicarles a sus hijos los riesgos de fumar, la presión que ejerce el grupo y la inexperiencia de hacer algo desconocido sin medir las consecuencias, son ejemplos de las causas que determinan las cifras lamentables de una situación que hoy estamos conociendo.

En este episodio, partiendo de una problemática real y significativa, se genera el interés en los estudiantes por expresar sus opiniones ante un fenómeno que sucede con personas que son contemporáneos a ellos. En la interacción didáctica, el docente formula una pregunta ( $\mathrm{Ccg}$ ) seguida de una instrucción (Ccd). Los alumnos se toman un tiempo en silencio para pensar en sus respuestas (Ccd) y luego participan uno por uno emitiendo sus opiniones (Ccd). El docente los escucha, acepta sus ideas (Ccd), las reconoce por medio de repeticiones literales ( $\mathrm{Ccg}$ ) con el ejercicio de una escucha abierta que transmite el aceptar las ideas (Ccd) y, finalmente, efectúa reelaboraciones ( $\mathrm{Ccg}$ ) para complementar la información de las intervenciones.

En las entrevistas, los docentes declaran que recurrir a ejemplos de noticias actuales y experiencias cotidianas es un recurso didáctico que usan porque les facilita contextualizar situaciones significativas y experiencias cotidianas para conectarlas con el desarrollo de capacidades y contenidos curriculares. De esta forma, se evidencia que el aprendizaje es inseparable del contexto donde se adquiere y utiliza (Coll y Sánchez, 2008). 
En importante señalar también que, a través de la secuencia de turnos en la conversación, códigos y criterios compartidos en la participación, se evidencian las reglas implicadas en la comunicación en el aula (Cubero et al., 2008).

\section{Generar interés y disposición como condición para el aprendizaje}

Consiste en que los estudiantes se involucren en las situaciones significativas al tener claro qué se pretende de ellas y al sentir que, con ello, se cubre una necesidad o un propósito de su interés. Así, la autonomía y la motivación para el aprendizaje se favorecen a medida que puedan participar en la planificación de lo que se realizará, y también se promueve la responsabilidad, al conocer los criterios mediante los cuales se evaluarán sus respuestas y, más aún, si les es posible mejorarlas en el proceso (Minedu, 2016).

Seleccionamos un episodio de una interacción en la sesión de clase de Ciencia, tecnología y ambiente en tercer año de secundaria:

Docente: A ver, Wilter, ¿cómo se llama tu juego y en qué consiste?

Wilter: Mi juego se llama "El rompe-tablero químico", porque es un rompecabezas y en él vamos a ubicar los elementos químicos.

Docente: ¿Con qué vas a empezar?

Wilter: Nos dan un elemento "H", que es hidrógeno, y tengo que ubicarlo en el rompecabezas.

Docente: ¿En qué grupo se ubica el hidrógeno?

Wilter: En el grupo uno.

Docente: Bien, el hidrógeno se encuentra en el grupo uno. ¿El sodio en qué grupo va?

Wilter: Uno.

Docente: El sodio también se encuentra en el grupo uno, ¿y en qué periodo está?

Wilter: Tres.

Docente: ¿Aprendemos mejor sobre química cuando realizamos juegos como el que nos ha mostrado Wilter?

Marlon: Sí, porque es más divertido y concreto. [El docente extiende las manos y las mueve en señal de aceptación.]

Yuri: Es más fácil de memorizar y a la vez es más divertido y dinámico.

Docente: Muy bien, estamos aprendiendo, jugando química. Ahora, yo quiero invitarlos a revisar en YouTube un video de unos jóvenes cantando rap sobre los elementos químicos. Hay mucha creatividad para aprender y espero que visiten este video para luego comentarlo en clase.

Este fragmento de la sesión de clase se contextualiza en una actividad lúdica en la que los estudiantes están compartiendo los juegos sobre química que elaboraron con anterioridad. La interacción muestra al docente formulando preguntas concretas (Ccg) y, de inmediato, recibe las respuestas de un alumno (Ccd), en referencia a la ubicación de los símbolos químicos que organizó en su rompecabezas. Estas respuestas son aceptadas y confirmadas por el docente a través de repeticiones literales (Ccg) y gestos ilustrativos, como el movimiento de las manos extendidas a 
ambos lados, que refuerzan las intervenciones (Ccg). Finalmente, el docente realiza un metaenunciado ( $\mathrm{Cg}$ ) por medio del cual invita a los alumnos a ver un video sobre una actividad musical relacionada con el contenido curricular.

En las entrevistas, un docente afirma que "las preguntas tienen que ser planteadas desde el principio de una manera dialogada, buscando en sí el interés del estudiante" y otro docente expresa que "la pregunta es básica para explorar qué es lo que sabe el alumno". Sin embargo, en el estudio de González et al. (2017) se demuestra la frecuencia de preguntas cerradas y literales que esperan, por lo general, una respuesta única por parte del estudiante, la cual puede ser un dato concreto o específico. Castellá et al. (2007) explican que los docentes acostumbran integrar en las explicaciones preguntas sobre cuestiones concretas para que los estudiantes puedan responder con facilidad, mantener la atención y, así, comprobar el grado de seguimiento.

\section{Aprender haciendo}

Esta orientación invoca que aprender y hacer son procesos indesligables, en los cuales la actividad y el contexto son clave para el aprendizaje. Ello implica que los estudiantes "pongan en juego sus capacidades reflexivas y críticas, aprendan a partir de su experiencia, identificando el problema, investigando sobre él, formulando alguna hipótesis viable de solución, comprobándola en la acción, entre otras acciones" (Minedu, 2016, p. 172).

Seleccionamos un episodio de una interacción en la sesión de clase de Ciencia, tecnología y ambiente en segundo año de secundaria. El docente pide a su alumno Sebastián que ejemplifique el tipo de movimiento recto uniforme, explicado minutos antes en clase. El alumno se desplaza por la pizarra con el plumón en la mano, da un paso y traslada el plumón de un punto a otro. Luego, da otro paso y vuelve a trasladar el plumón de un punto a otro. Finalmente, da un último paso y repite la acción. En la experiencia, explica:

Sebastián: Un cuerpo es un móvil, va de un punto a otro, de forma recta y sin acelerar ni bajar su velocidad; va solamente a un ritmo.

Docente: ¿Qué tipo de movimiento será?

Alumnos: Movimiento rectilíneo.

Docente: Movimiento rectilíneo uniforme. Es igual como yo. Estoy parado, empiezo a moverme y voy a un solo ritmo de un lugar a otro. No estoy acelerando, ni estoy bajando. ¿Qué movimiento será? [El docente se ubica delante de la puerta y empieza a dar pasos de forma lenta. Expresa gestos con la mirada para validar las intervenciones.]

Alumnos: Movimiento rectilíneo...

Docente: Movimiento rectilíneo uniforme.

Partiendo el docente de un marco específico de referencia (Ccg) por el cual quiere darle continuidad a un contenido curricular desarrollado anteriormente, le pide a un estudiante que explique un ejemplo graficado en la pizarra. En seguida, el alumno responde a la actividad (Ccd) y el docente reformula el ejemplo (Ccg), usa repeticiones literales (Ccg), gestos faciales, mirada fija y apertura exagerada de los ojos (Ccg) para indicar que la respuesta es acertada (Ccd). 
En la entrevista, un docente de ciencias explica:

De acuerdo con el enfoque de la indagación científica, las respuestas que me dan los estudiantes las anoto como tal y no las puedo corregir, porque estamos hablando de ciencia. Entonces, las respuestas que el alumno me da son muy importantes. Así estén erradas, sean medias verdades, hay que anotarlas tal cuales; de tal manera que, en el proceso del aprendizaje, al plantearse una hipótesis y entrar a un experimento, ellos solos tendrán que darse cuenta de que sus hipótesis eran verdaderas o falsas. Es allí donde el alumno aprende.

Este episodio interactivo caracteriza una frecuencia de respuestas concretas de los estudiantes ante preguntas concretas del docente. Sin embargo, si en dos oportunidades los estudiantes respondieron "movimiento rectilíneo" y el docente hizo hincapié en la característica faltante "uniforme", era necesario destacar y explicar aquella característica que no había sido aún comprendida por los estudiantes.

\section{Partir de los saberes previos}

Consiste en recuperar a través de preguntas o tareas los conocimientos, concepciones, representaciones, vivencias, creencias, emociones y habilidades adquiridos antes por el estudiante respecto a lo que se propone aprender al enfrentar la situación significativa. A través de estos saberes previos, el estudiante entra en contacto con el nuevo conocimiento, y el docente puede hacerse una idea sobre cuánto ya sabe o domina de lo que él quiere enseñarle (Minedu, 2016).

Seleccionamos un episodio de una interacción en la sesión de clase de Comunicación en segundo año de secundaria:

Docente: Entonces, como ya habíamos hablado del guion teatral, con todas sus características, ¿verdad?, el propósito de la clase de hoy es analizar un texto dramático. Les pregunto: ¿la lectura que tienen en la página 212 de su libro será un guion teatral?

Estudiantes: Sí.

Docente: ¿Cómo reconocemos que es un guion teatral?

Julián: Porque tiene personajes que realizan una acción.

Docente: Que acción realizan... ¿Cuántos personajes hay?

Estudiantes: Siete.

Docente: Siete, y cada uno tiene su diálogo. ¿El narrador tiene diálogo?

Estudiantes: No.

Docente: ¿En un guion teatral como este, el narrador figura como personaje?

Estudiantes: No.

Docente: Muy bien, los personajes son aquellos que realizan una acción a través del diálogo. Muy bien, eso es lo que vamos a hacer hoy, analizar un texto dramático entonces.

En este diálogo, el docente parte de un metaenunciado (Ccg), que consiste en mencionar el propósito de la clase y las acciones que se desarrollarán; en seguida, formula preguntas (Ccg) y explica las relaciones que se establecen entre los nuevos contenidos y los anteriores. En ese proceso, los estudiantes responden las preguntas (Ccd), el docente moviliza y refuerza los saberes previos, y da una instrucción (Ccd) para que se desarrolle la primera actividad. 
En este episodio, los estudiantes participan respondiendo preguntas concretas, pero solo lo hacen cuando el docente las formula, es decir, no se evidencian participaciones espontáneas. Entendiendo que había un conocimiento previo sobre el guion teatral, las respuestas pudieron dar pie a acciones de retroalimentación (comentarios, ejemplos, preguntas, discusiones) antes del desarrollo de la siguiente actividad, que era más compleja: el análisis del texto dramático. De acuerdo con Coll (1988), mientras más relaciones el estudiante pueda realizar con sus saberes previos, el aprendizaje será más significativo.

\section{Construir el nuevo conocimiento}

Requiere que el estudiante maneje, además de las habilidades cognitivas, la información, los principios, las leyes, los conceptos o teorías que le ayudarán a entender y afrontar los retos planteados dentro de un determinado campo de acción. Importa que logre un dominio aceptable de conocimientos para que los pueda transferir y aplicar de manera pertinente en situaciones concretas (Minedu, 2016).

Seleccionamos un episodio de una interacción en la sesión de clase de Arte en cuarto año de secundaria:

Docente: Bien. Primero, en nuestro boceto, ¿qué vamos a identificar? Vamos a identificar los planos. ¿Alguna vez les han hablado de planos?

Alumnos: Sí. De arquitectura. De la casa.

Docente: Ya, de arquitectura. En la pintura, los planos se refieren a los espacios que se representan en nuestro trabajo. Por ejemplo, este es un bodegón que contiene tres planos. Primer plano, todo lo que está delante de esta cabeza que está rodando como una esfera. Segundo plano, la vegetación que está atrás. Tercer y último plano, todo lo que queda en el horizonte. ¿Sí? Por ejemplo, yo me voy a tomar una foto, ¿qué es lo que queda atrás?

Alumnos: El ambiente, la pizarra.

Docente: La pizarra, ¿verdad? Sin embargo, en primer plano va a estar mi figura, mi persona.

En este diálogo, hay una acción específica referida a la identificación de planos en un boceto. Para ello, es fundamental la formulación de preguntas (Ccg) y, con base en las respuestas, se dan las repeticiones literales (Ccg) como un mecanismo que usa el docente para validar las intervenciones de los alumnos, las cuales van acompañadas de una inclinación positiva de la cabeza en señal de aceptación (Ccd). Asimismo, el docente aprovecha las intervenciones para añadir una información extra (Ccg).

Un elemento crucial en las interacciones es la retroalimentación (González et al., 2017) que debe ser generada por las preguntas que formule el docente, las respuestas de los estudiantes, y viceversa. Sin embargo, pareciera que el docente espera respuestas determinadas que, de alguna manera, "acierten" lo que está esperando.

\section{Aprender del error o el error constructivo}

La orientación considera que el error puede ser empleado de forma constructiva, como una oportunidad de aprendizaje, y propiciar la reflexión y revisión de los 
diversos productos, tanto del profesor como del estudiante. "El error requiere diálogo, análisis, una revisión cuidadosa de los factores y decisiones que llevaron a él. Esta forma de abordarlo debe ser considerada tanto en la metodología como en la interacción continua profesor estudiante" (Minedu, 2016, p. 172).

Seleccionamos un episodio de una interacción en la sesión de clase de Matemática en primer año de secundaria:

Docente: ¿Qué era el complemento de un ángulo? Si yo les decía que la puerta está entreabierta y medía sesenta grados. Y ustedes, ¿qué hicieron para determinar qué era treinta grados? ¿Qué hicieron?

José: Una ecuación.

Docente: ¿Estás seguro de lo que dices? No fue una ecuación, fue una operación más sencilla. [El docente hace un gesto de sorpresa abriendo exageradamente los ojos, mientras realiza movimientos circulares de las manos.]

José: ¿Resta?

Docente: Una resta. Entonces, ¿qué es el complemento de un ángulo? Es lo que le faltaba.

José: La diferencia.

Docente: La diferencia. Es lo que le faltaba al ángulo, a sesenta grados, para ser igual a noventa grados, ¿no? Ese es el complemento de un ángulo.

Partiendo de la idea de que los errores no pueden considerarse "fallas" en el aprendizaje, este diálogo muestra la formulación de una pregunta del docente (Ccg) sobre un tipo de operación matemática, que destaca el procedimiento realizado. Sin embargo, la intervención de un alumno (Ccd), a través de una respuesta concreta, es equivocada. Ante esa situación, el docente insiste y ofrece una reformulación (Ccg), mediante la cual induce a una respuesta (Ccd) acompañada de gestos con los ojos y las manos (Ccg), que logrará la revisión de la operación por parte del estudiante y la obtención de la respuesta correcta (Ccd).

En las entrevistas, un docente declara que "cuando es necesario corregir, se le ayuda al estudiante para que él mismo se dé cuenta del error, incluso para que él mismo sea quien se corrija”. Otra docente manifiesta que "por más respuesta inválida que dé el alumno, hay que tratar de orientarlo y apoyarlo, porque hay que destacar ese deseo de participar". Estas afirmaciones convergen con Astolfi (2003), quien plantea que los errores no deberían concebirse como "fallos" del aprendizaje, sino más bien representan un medio para enseñar y aprender del error.

\section{Generar el conflicto cognitivo}

Consiste en plantear un reto cognitivo que le resulte significativo al estudiante y cuya solución permita desarrollar sus diversas capacidades. Al trabajar con una información o un comportamiento que contradice y discute sus creencias, se produce una desarmonía en el sistema de ideas, creencias y emociones de la persona, que, si involucra su interés, puede motivar la búsqueda de una respuesta, lo que abre paso a un nuevo aprendizaje (Minedu, 2016).

En las sesiones de clases observadas no se localizó un episodio en el que se evidencie esta orientación; sin embargo, en las entrevistas, un docente de 
Ciencia, tecnología y ambiente compartió una experiencia de lo que para él significa generar el conflicto cognitivo en el aula:

Por ejemplo, ayer estábamos hablando de cómo ocurre la diploidía en los humanos, y un alumno me dice "profesor, usted está hablando de la diploidía en los humanos, pero ¿si fuera más allá de la diploidía?". Yo le digo "o sea, tú me quieres dar a entender que un ser vivo que tiene número 46, pueda tener 50, 60 o 100 . Esa pregunta es importante porque podríamos definir que a lo más un ser vivo, el ser humano en este caso presentaría dos cromosomas de más. Pero no sería una doble diploidía, sino una poliploidía. Me parece importante tu pregunta, porque me indica que estás analizando. Así te podrían decir que las plantas son poliploides, y la ventaja de las plantas poliploides es que son más fuerte, por ejemplo".

La pregunta del estudiante sobre diploidía y la interpretación y posterior ejemplificación que hace el docente sobre ella plantea la importancia de un conocimiento situado, producto de la actividad y la cultura en la que se desarrolla (Díaz, 2006). En ese sentido, se resalta la transferencia del conocimiento a situaciones concretas, pues al final estas tienen que lograr ser aplicadas en la vida cotidiana.

\section{Mediar el progreso de los estudiantes de un nivel de aprendizaje a otro superior}

La mediación del docente durante el proceso de aprendizaje supone acompañar al estudiante hacia un nivel inmediatamente superior de posibilidades (zona de desarrollo próximo) respecto a su nivel actual (zona real de aprendizaje), por lo menos hasta que el estudiante pueda desempeñarse bien de manera independiente. De este modo, es necesaria una conducción cuidadosa del proceso de aprendizaje, en la cual la atenta observación del docente permita al estudiante realizar tareas con distintos niveles de dificultad (Minedu, 2016).

Seleccionamos un episodio de una interacción en la sesión de clase de Matemática en segundo año de secundaria:

Docente: Bien, ya habíamos hecho una ficha de ecuaciones, ¿verdad? Les había dicho qué es una ecuación, ¿no? ¿Qué era una ecuación?

Juan: Una igualdad algebraica.

Docente: Es una igualdad algebraica. Por ejemplo, cuando nosotros hablamos de un número, si no sabemos cuál es el número, ¿cómo lo expresamos?

Algunos estudiantes: Equis. [La docente camina mientras realiza las preguntas. Los estudiantes responden desde sus posiciones. Ahora, la docente procede a pegar el papelógrafo en la pizarra.]

Docente: Les voy a poner este ejemplo: el doble de la edad de Carlos, aumentado en tres, es igual a treinta y tres. ¿Cuántos años tiene Carlos? A ver, ¿alguien lo quiere plantear en la pizarra? Yo les estoy colocando el enunciado en forma verbal y ustedes tienen que traducirlo en forma simbólica y viceversa ¿no? [Los alumnos no responden, hay silencio...]

Docente: Sea la edad de Carlos "x", ya que no conocemos cuántos años tiene, entonces el doble de su edad se representaría por " $2 x^{\prime}$; luego, si este doble está aumentado en tres sería: 2x más 3 igual 33. ¿Cuántos años tiene Carlos actualmente? Tiene quince años. Ahora que ya se conoce que Carlos tiene 15 años, ¿cuántos años tendrá dentro de dieciocho años?

María: Treinta y tres.

Docente: Treinta y tres. 
Docente: Hoy día vamos a ver, entonces, la traducción de enunciados verbales a enunciados simbólicos y viceversa.

Durante la interacción, el docente se esfuerza por activar los saberes previos al formular preguntas sobre contenidos de ecuaciones de una clase anterior ( $\mathrm{Ccg}$ ). El ejercicio se materializa en un esquema escrito en un papelógrafo y pegado en la pizarra, el cual sirve de guion para asegurar el orden lógico de la operación y la explicación del docente. A través de este recurso visual, el docente formula preguntas $(\mathrm{Ccg})$ y recibe respuestas inmediatas de los estudiantes (Ccd). Por último, el docente realiza un metaenunciado $(\mathrm{Ccg})$ al contextualizar la actividad que a continuación se llevará a cabo.

En este episodio, el docente desarrolla actividades que parten de la evocación de la información para trabajar contenidos que exigen cierto nivel de abstracción en la representación simbólica de una actividad algebraica. De esa forma, a través de la retroalimentación, orienta a los estudiantes a la comprensión de habilidades complejas.

\section{Promover el pensamiento complejo}

En el enfoque por competencias se busca que los estudiantes aprendan a analizar la realidad que los desafía de una manera integrada y no fragmentada, y relacionen sus distintas características a fin de explicarla. Eso implica que las diferentes disciplinas se complementen para comprender más cabalmente los problemas y desafíos de la realidad en sus múltiples dimensiones (Minedu, 2016).

En las sesiones de clases no observamos un episodio que haga evidente esta orientación; sin embargo, en las entrevistas, un docente de matemática comparte una experiencia de lo que para él significa promover el pensamiento complejo en el aula:

Por ejemplo, nosotros tenemos un proyecto el cual ha sido dividido en subáreas, Álgebra, Aritmética, Geometría y Razonamiento matemático. Por ejemplo, cuando yo quiero entrar a Geometría, donde resolvemos problemas geométricos, tengo que hacer en Álgebra ecuaciones, porque entonces entro en Álgebra y después recién puedo entrar a Geometría para resolver problemas. 0 en el mismo Álgebra, ¿no? Por ejemplo, nos sirve para ver funciones, tengo que ver el plano cartesiano y de ahí recién puedo entrar a la función lineal, ecuación de segundo grado, valor absoluto, o ver las operaciones elementales para poder entrar, por ejemplo, a Razonamiento matemático, resoluciones numéricas, analogías para plantear un problema; ellos tienen que plantear su ecuación.

Tomando como referencia el enfoque por competencias (Minedu, 2016), que orienta una práctica pedagógica hacia la combinación de las capacidades (conocimientos, habilidades y actitudes), se puede entender que el proyecto matemático expresado por el docente se nutre del aporte de cada una de las áreas.

\section{DISCUSIÓN Y CONCLUSIONES}

Partiendo de un objeto de estudio, como son las orientaciones pedagógicas para el desarrollo de competencias en el aprendizaje (Minedu, 2016), nuestra investigación se propuso identificar y analizar las relaciones en las interacciones didácticas; confirmamos evidencias en la complementariedad e interdependencia de las categorías cognitivas (Fernández y Cuadrado, 2008) y conductuales (Velasco, 2007). 
Nuestro estudio muestra episodios interactivos de sesiones de clase, en los cuales se diagnostica la presencia de siete de las diez orientaciones pedagógicas para el desarrollo de competencias propuestas por el Minedu (2016). Estas orientaciones se refieren a: partir de situaciones significativas; generar interés y disposición como condición para el aprendizaje; aprender haciendo; partir de los saberes previos; construir el nuevo conocimiento; aprender del error o el error constructivo; y mediar el progreso de los estudiantes de un nivel de aprendizaje a otro superior.

En esos episodios se conjugan las categorías cognitivas y conductuales como constructos que vinculan cognición, conducta y desempeño (Velasco, 2007), definidas como interacciones didácticas, las cuales emergen y toman cuerpo a medida que se despliegan las actuaciones interrelacionadas (Villalta y Martinic, 2009).

Sin embargo, en esos episodios predomina la presencia de un número mayor de interacciones cognitivas en comparación con las interacciones conductuales. Velasco (2007) señala que en la estructura de la interacción hay intencionalidades instantáneas (espontáneas o no), por lo cual podríamos interpretar, a través de esta evidencia, que en las prácticas docentes observadas la cognición tiene mayor peso que la conducta.

Las categorías cognitivas en las participaciones docentes, identificadas en orden de mayor frecuencia de aparición, son: formulación de preguntas, repeticiones literales, reformulaciones, metaenunciados, gestos ilustrativos, reelaboraciones y marco específico de referencia. Estas categorías reflejan una relación entre los mecanismos comunicativos, verbales y no verbales que desarrolla el docente y el proceso cognitivo que se demanda de los alumnos. Las categorías cognitivas no identificadas fueron: marco social de referencia, autorreformulaciones y resúmenes.

Las categorías conductuales en las participaciones docentes, mencionadas en orden de mayor frecuencia de aparición, son: acepta las ideas u opiniones; estimula, apoya, acompaña, aprueba; instruye, expresa conocimiento, responde, aclara; corrige e induce a la respuesta. No obstante, la única categoría conductual de los estudiantes, encontrada en todos los episodios interactivos, es la de responde, expone o expresa conocimiento. Esos resultados evidencian mayor frecuencia de intervención de los docentes en relación con la participación de los estudiantes, a quienes habría que denominar "oyentes" antes que educandos (Velasco y Alonso, 2008).

Dos orientaciones pedagógicas para el desarrollo de competencias propuestas por el Minedu (2016) no fueron ubicadas en los episodios interactivos. Estas son generar el conflicto cognitivo y promover el pensamiento complejo; sin embargo, los docentes declararon en las entrevistas el sentido de la acción de su práctica, a través de las cuales explican cómo toman en cuenta ambas orientaciones. Sobre el desarrollo del pensamiento complejo, González et al. (2017) demuestran en su estudio que ese nivel de pensamiento se encuentra ausente en la práctica docente y que las actividades llevadas a cabo se localizan en el nivel de la evocación o identificación de la información específica.

Una orientación pedagógica para el desarrollo de competencias no fue localizada ni en los episodios ni en las entrevistas. Esta promueve el trabajo cooperativo. El Minedu (2016) explica que esta orientación busca que los estudiantes hagan frente a una situación retadora en la que complementen sus diversos conocimientos, habilidades y 
destrezas de una forma cooperativa y colaborativa. Sin embargo, en el estudio, solo se constataron episodios en los que el docente dejaba un cuestionario y los estudiantes organizados en grupos se distribuían las preguntas para luego compartir las respuestas y escribirlas en sus cuadernos. En este caso podemos confirmar la disonancia entre una orientación que es intencionalmente participativa y una práctica que genera el desarrollo de productos individuales por parte de los estudiantes.

Para concluir, las aproximaciones de este estudio pretenden mostrar evidencias que contribuyan al análisis de lo que ocurre en el contexto específico del aula, caracterizado por procesos de intercambio en los que existen diferentes formas y estilos de comunicación verbal y no verbal del docente y los estudiantes. Reconocemos que debe existir una correspondencia entre el análisis de la interacción didáctica y tres aspectos de la práctica docente: una estructura dialógica en la sesión de clase que incluya turnos de actuación y mediación equitativos entre docentes y estudiantes con finalidades claras; una planificación, ejecución y evaluación progresiva del enfoque basado en el aprendizaje por competencias; una continua autoevaluación docente con elementos proporcionados por los órganos educativos, que implique encontrarle sentido al cambio de algunas prácticas pedagógicas habituales.

Finalmente, el análisis del discurso basado en las relaciones cognitivas y conductuales de las prácticas pedagógicas se podría aplicar en distintos niveles, contextos y modalidades conformados por grupos interactivos en la enseñanza y aprendizaje. De ese modo, queda claro que las acciones comunicativas intencionadas que se desarrollan en el aula entre docente y estudiantes encierran, implícita o explícitamente, un tipo de aprendizaje y todo lo que es posible aprender se constituye en una interacción.

\section{REFERENCIAS BIBLIOGRÁFICAS}

Astolfi, J. (2003). El "error", un medio para enseñar. Sevilla: Diada Editorial.

Castellá, J., Comelles, S., Cros, A. y Vilá, M. (2007). Entender (se) en clase. Las estrategias comunicativas de los docentes bien valorados. Barcelona: Editorial Graó.

Coll, C. (1988). Significado y sentido en el aprendizaje escolar. Reflexiones en torno al concepto de aprendizaje significativo. Infancia y aprendizaje, vol. 11, núm. 41, pp. 131-142. Recuperado de https://www.tandfonline.com/doi/ab s/10.1080/02103702.1988.10822196.

Coll, C. y Sánchez, E. (2008). Presentación. El análisis de la interacción alumnoprofesor: líneas de investigación. Revista de Educación, núm. 346, pp. 15-32. Recuperado de http://www.revistaeducacion.mec.es/re346/re346_01.pdf

Cuadrado, I. y Fernández, I. (2008). Adaptaciones cognitivo-lingüísticas del profesor a los comportamientos comunicativos de los alumnos. Revista de Educación, núm. 345, pp. 301-328. Recuperado de https://dialnet.unirioja.es/ servlet/articulo?codigo $=2506139$.

Cubero, R., Cubero, M., Santamaría, A., De la Mata, M., Carmona, I. y Prados, M. (2008). La educación a través de su discurso. Prácticas educativas y construcción discursiva del conocimiento en el aula. Revista de Educación, núm. 346, pp. 71-104. Recuperado de https://idus.us.es/xmlui/handle/11441/31864. 
Díaz, A. (2006). El enfoque de competencias en la educación. ¿Una alternativa o un disfraz de cambio? Perfiles Educativos, vol. 28, núm. 111,pp. 7-36. Recuperado de http://www.iisue.unam.mx/perfiles/articulo/2006-111-el-enfoquede-competencias-en-la-educacion-una-alternativa-o-un-disfraz-de-cambio. pdf

Díaz, L. y Ochoa, J. (2007). Construcción y reconstrucción de saberes en educación. Ponencia presentada en el XIX Encuentro Nacional y V Internacional de Investigadores en Educación, en Santiago de Chile.

Forero-Saenz, A. (2008). Interacción y discurso en la clase de matemática. Universitas Psychologica, vol. 7, núm. 3, pp. 787-805. Recuperado de http://revistas.javeriana.edu.co/index.php/revPsycho/article/view/392

González, N., Eguren, M. y De Belaunde, C. (2017). Desde el aula: una aproximación a las prácticas pedagógicas del maestro peruano. Lima: Instituto de Estudios Peruanos, serie Educación, 13.

Guerrero, G. (2018). Estudio sobre la implementación del Currículo Nacional de la Educación Básica en instituciones públicas focalizadas. Lima: GRADE-Proyecto FORGE. Recuperado de http://www.grade.org.pe/forge/descargas/CurriculoNacional.pdf.

Luengo, J., Luzón, A. y Torres, M. (2008). El enfoque por competencias en el desarrollo de políticas de formación del profesorado. Entrevista a Claude Lessard. Profesorado. Revista de Currículum y Formación del Profesorado, vol. 12, núm. 3, pp. 1-16. Recuperado de https://www.ugr.es/ recfpro/rev123ART5.pdf

Mares, G., Guevara, Y., Rueda, E., Rivas, 0. y Rocha, H. (2004). Análisis de las interacciones maestra-alumnos durante la enseñanza de las ciencias naturales en primaria. Revista Mexicana de Investigación Educativa, vol. 9, núm. 22, pp. 721-745. Recuperado de http://www.comie.org.mx/v1/revista/portal. php?idm=es\&sec $=$ SC03\&\&sub=SBB \&criterio=ART00151

Martinic , S., Vergara, C. y Huepe, D. (2013). Uso del tiempo e interacciones en la sala de clases. Un estudio de casos en Chile. Pro-Posições, vol. 24, núm. 1 (70), pp. 123-135. Recuperado de https://periodicos.sbu.unicamp.br/ojs/index. php/proposic/article/view/8642665.

Ministerio de Educación del Perú (Minedu) (2016). Currículo nacional de la educación básica. Lima, Perú.

Ministerio de Educación del Perú (Minedu) (2012). Marco del buen desempeño docente. Lima, Perú.

Perrenoud, P. (2008). Construir las competencias: ¿es darle la espalda a los saberes? Red U. Revista de Docencia Universitaria, pp. 1-8. Recuperado de http://revistas.um.es/redu/article/view/35261

Rogoff, B. (1993). Aprendices del pensamiento: el desarrollo cognitivo en el contexto social. Barcelona: Paidós.

Sánchez, E., García, R., Castellano, N., De Sixte, R., Bustos, A. y García-Rodicio, H. (2008). Qué, cómo y quién: tres dimensiones para analizar la práctica educativa. Cultura y Educación, vol. 20, núm. 1, pp. 95-118. Recuperado de https:// www.tandfonline.com/doi/abs/10.1174/113564008783781431?journalCo de=rcye20.

Tapia J. y Cueto, S. (2017). El apoyo de FORGE al desarrollo del Currículo Nacional de Educación Básica del Perú. Informe final. Lima: GRADE-Proyecto FORGE. Recuperado de http://www.grade.org.pe/forge/descargas/ Sistematizaci\%C3\%B3n\%20Curriculo.pdf. 
Varguillas, C. (2006). El uso de Atlas.ti y la creatividad del investigador en el análisis cualitativo de contenido. Laurus, Revista de Educación, pp. 73-87. Recuperado de http://132.248.192.201/seccion/bd_iresie/iresie_busqueda. php?indice=autor\&busqueda=VARGUILLAS, \%20CARMEN\&par=todos\&a inicial=2004\&a_final=2009\&sesion=2018-08-15\%2014:38:25\&formato=

Velasco, A. (2007). Un sistema para el análisis de la interacción en el aula. Revista Iberoamericana de Educación, vol. 42, núm. 3, pp. 1-12. Recuperado de https://rieoei.org/RIE/article/view/2421.

Velasco, A. y Alonso, L. (2008). Sobre la teoría de la educación dialógica. Revista Educere, vol. 12, núm. 42, pp. 461-470. Recuperado de http://www.saber.ula. ve/handle/123456789/26301

Villalta, M., Assael, C. y Martinic, S. (2013). Conocimiento escolar y procesos cognitivos en la interacción didáctica en la sala de clase. Perfiles Educativos, vol. 35, núm. 141, pp. 84-96. Recuperado de http://www.iisue.unam.mx/perfiles/articulo/2013-141-conocimiento-escolar-y-procesos-cognitivos-en-lainteraccion-didactica-en-la-sala-de-clase.pdf

Villalta, M., Martinic, S. y Guzmán, M. (2011). Elementos de la interacción didáctica en la sala de clase que contribuyan al aprendizaje en contexto social vulnerable. Revista Mexicana de Investigación Educativa, vol. 16, núm. 51, pp. 1137-1158. Recuperado de http://www.comie.org.mx/v1/revista/portal.ph p?idm $=$ es\&sec $=$ SC03\&\&sub $=$ SBB \&criterio $=$ ART51005

Villalta, M. y Martinic, S. (2013). Interacción didáctica y procesos cognitivos. Una aproximación desde la práctica y discurso del docente. Universitas Psychologica, vol. 12, núm. 1, pp. 221-233. Recuperado de http://revistas.javeriana. edu.co/index.php/revPsycho/article/view/1325

Villalta, M. y Martinic, S. (2009). Modelos de estudio de la interacción didáctica en la sala de clase. Investigación y Postgrado, vol. 24, núm. 2, pp. 61-76. Recuperado de http://revistas.upel.edu.ve/index.php/revinpost/article/ view/920 